Incidencia del control interno en la gestión administrativa de las IES. Caso: Departamento de Pastoral, Universidad Politécnica Salesiana

Incidence of internal control in the administrative management of IES. Case: Departamento de Pastoral, Universidad Politécnica Salesiana

\author{
Erika Priscila Zamora Cabrera \\ ezamorac@psg.ucacue.edu.ec \\ Universidad Católica de Cuenca, Cuenca \\ Ecuador \\ https://orcid.org/0000-0001-9085-172X \\ Cecilia Ivonne Narváez Zurita \\ inarvaez@ucacue.edu.ec \\ Universidad Católica de Cuenca, Cuenca \\ Ecuador \\ https://orcid.org/0000-0002-7437-9880 \\ Juan Carlos Erazo Álvarez \\ jcerazo@ucacue.edu.ec \\ Universidad Católica de Cuenca, Cuenca \\ Ecuador \\ https://orcid.org/0000-0001-6480-2270
}

Recibido: 1 de septiembre de 2019

Aprobado: 30 de septiembre de 2019

\title{
RESUMEN
}

Toda organización se plantea objetivos a cumplir a corto y largo plazo; para ello se ayuda de diferentes procesos en los cuales es necesario ejecutar controles que permitan verificar su correcto funcionamiento. Esta investigación se basó en el modelo COSO III para establecer procedimientos de control interno que permitan al proceso de Tutorías Académicas aportar de manera correcta con uno de los objetivos de la Universidad Politécnica Salesiana que es el garantizar la permanencia y titulación de todos sus estudiantes, para ello se utilizaron técnicas como encuestas, entrevistas, observación y análisis documental. Los resultados obtenidos demostraron que el proceso no cuenta con controles estratégicos en los puntos críticos, la institución no tiene manuales de funciones 
para este proceso, además el proceso se ejecutó de manera empírica por los estudiantes tutores. Con los procedimientos de control interno establecidos, el proceso se ejecuta de manera correcta y se evalúa continuamente.

Descriptores: Control interno; COSO; Gestión; Procedimientos; Tutorías académicas.

\begin{abstract}
Every organization sets goals to meet in the short and long term; To do this, it helps with different processes in which it is necessary to execute controls that allow to verify its correct operation. This research was based on the COSO III model to establish internal control procedures that allow the Academic Tutoring process to contribute correctly with one of the objectives of the Salesian Polytechnic University, which is to guarantee the permanence and qualification of all its students, to this used techniques such as surveys, interviews, observation and documentary analysis. The results obtained showed that the process does not have strategic controls at the critical points, the institution does not have function manuals for this process, in addition the process was executed empirically by the student tutors. With the established internal control procedures, the process is executed correctly and is continuously evaluated.
\end{abstract}

Descriptors: Internal control; COSO; Management; Procedures; Academic tutoring.

\title{
INTRODUCCIÓN
}

La educación superior en el Ecuador ha dado un giro desde el 2010 cuando inició la evaluación a las universidades en busca de una mejor calidad, lo que ha llevado al cierre de varias Instituciones de Educación Superior (IES) y a cambios en infraestructura, planta docente y metodologías de enseñanzas, en otras (SENESCYT, 2014). Adicionalmente, se inicia un mecanismo de evaluación a los estudiantes que deseen ingresar a instituciones públicas, quienes al aprobar el Examen Nacional de Educación Superior (ENES) son ubicados en IES privadas con convenio. Éstas deben garantizar el ingreso, permanencia y graduación de los estudiantes, por tal motivo la Universidad Politécnica Salesiana ha desarrollado un programa de acompañamiento, el mismo que se da en los 4 primeros ciclos y para ello es necesario tener un sistema determinado de aplicación que ayude a responder la pregunta ¿cómo evaluar el cumplimiento y aplicación de los procedimientos, normas legales y universitarias vigentes en el área de Tutorías 
Académicas de la Universidad Politécnica Salesiana sede Cuenca? (Universidad Politécnica Salesiana, 2015).

En este sentido, se realiza una revisión de la literatura contemporánea con antigüedad de 10 años en donde se puede evidenciar la importancia que tiene para una institución establecer un sistema de control interno, pues éste ayudará a verificar el cumplimiento de objetivos a corto y largo plazo, también controlar el uso de los recursos, anticiparse a acontecimientos futuros de tal manera, que la Dirección tome decisiones oportunas como una reestructuración de los procesos (Isaza, 2014; Benavides, Narváez, Erazo y Ordoñez, 2019). Por lo expuesto, el presente artículo tiene por finalidad establecer procedimientos de control interno para el departamento de Pastoral de la Universidad Politécnica Salesiana sede Cuenca.

\section{Sistemas de control en las organizaciones}

En toda organización existe un departamento administrativo, que es el cual ejerce el control dentro de la empresa; para esto se establece procedimientos, estrategias para saber la situación de la organización, sistematizar funciones, establecer el logro de objetivos, cumplir con sus actividades de manera eficiente, y determinar si se está cumpliendo con las políticas de la misma. Por lo tanto, la administración establece controles en las diferentes áreas de la organización buscando evaluar el cumplimiento de las metas planteadas de acuerdo con las leyes aplicables (Estupiñán y Niebel, 2015).

El control interno es un proceso compuesto por varias actividades que lo realizan todas las personas que laboran en la organización con el fin de proporcionar seguridad razonable en el cumplimiento de objetivos (Santillana, Sistemas de Control Interno, 2015). Así mismo, Blanco (2012), Mantilla (2013) y Mendívil (2016) afirman que el control interno es un proceso en el que participa la junta directiva, la gerencia, el personal y la red de procesos, está diseñado para dar seguridad razonable en el logro de los objetivos, buscando que las operaciones sean efectivas y eficiente, la información debe ser confiable y todas las actividades deben cumplir las leyes y regulaciones de ese momento. Entonces, se puede definir al control interno como un proceso que lo ejecutan todos los 
miembros de una organización con el fin de tener un control sobre la información y las actividades que se deben realizar para que la institución cumpla de manera correcta con sus objetivos.

En este sentido, el sistema control interno tiene algunos objetivos fundamentales como: proteger los insumos de la institución ante un posible riesgo que afecte a la información o recursos; garantizar que las actividades se ejecuten con eficiencia, eficacia y economía, ligado directamente con la misión y visión de la organización; vigilar que las actividades de cada departamento estén dirigidos al logro de los objetivos planteados por la empresa; certificar una correcta evaluación y seguimiento de los procedimientos, evaluando la eficacia en el logro de metas, la eficiencia en el uso de recursos, la efectividad para determinar si la organización es eficaz, eficiente y económica; también, busca asegurar la oportunidad y veracidad de la información, fijar y aplicar mecanismos para prevenir riesgos, además de verificar el cumplimiento de leyes y normas (Mantilla, 2013; Isaza, 2014; Gamboa, Puente, y Vera, 2016; Tapia, Guevara, Castillo, Rojas, y Salomón, 2016; López y Pesántez, 2017; Quinaluisa, Ponce, Muñoz, Ortega, y Pérez, 2018).

Santillana (2015) afirma que los objetivos del control interno tienen varias aristas como el sistema contable, la autorización, el procesamiento y clasificación de las transacciones, la verificación, evaluación y la salvaguarda física. Por otro lado, tienen cuatro objetivos específicos: motivar al cumplimiento de la normativa, promover la eficiencia operativa, garantizar la razonabilidad y confiabilidad de información, y; salvaguardar los activos y la documentación relevante.

Así también otros autores como Atehortua, Bustamante, y Valencia (2008) establecen que para las instituciones gubernamentales en Colombia la Ley les obliga a implementar un modelo de control interno, siendo así utilizado por ellos el modelo estándar en el cual deben cumplir principios que estan establecidos en las normas como son el autocontrol, la autorregulación y la autogestión, también se evidencia que no puede existir un modelo de control sin antes diseñar un modelo de gestión organizacional ya que la ISO 9001 señala que la gestión es la encargada de la dirección y por supuesto el control de la empresa. 
De acuerdo a estos autores los componentes del modelo son 3: el control estratégico, que reconoce a la empresa como un conjunto de componentes que se interrelacionan y a través del control se evidencia el cumplimiento de los objetivos estratégicos; el control de gestión, que establece los controles que se deberán implementar para prevenir los riesgos y conseguir los resultados esperados, también se establecen controles para la información y la comunicación; finalmente, el control de evaluación, que permite dar seguimiento a la eficacia de los controles contra los riesgos que se han identificado así como tambien el cumplimiento de los objetivos trazados.

La aplicación del control interno en una organización es muy importante, como ya se ha mencionado permite mejorar el uso de los recursos publicos o privados, se genera mayor responsabilidad en los involucrados en cada actividad y compromiso con la entidad para lograr las metas, esto también genera un ambiente de tranquilidad para los dueños de las empresas ya que con un sistema confiable de control interno se mitigan los riesgos. Es por ello que en el siguiente epigrafe se definirá algunos modelos de control interno que han sido aplicados en distintas organizaciones.

\section{Los modelos de Control Interno}

Modelo COCO, este nace a partir del COSO I pues se necesitaba establecer un modelo de más fácil comprensión, por ello, un comité canadiense se ha encargado de la construcción de este modelo, estableciendo 20 criterios de evaluación agrupados según los objetivos, el compromiso, la aptitud, la evaluación y el aprendizaje; en dónde, los objetivos se deben dar a conocer a todos los miembros de la empresa, de igual manera establecer los riesgos que impidan su cumplimiento; el compromiso que debe tener cada trabajador debe estar directamente ligado con los valores éticos de la empresa, deberá definirse la autoridad y sus responsabilidades fomentando un ambiente de confianza y que la información fluya (Estupiñán y Niebel, 2015; Quinaluisa, Ponce, Muñoz, Ortega, y Pérez, 2018).

En cuanto a la aptitud, los integrantes de la organización deben contar con habilidades, conocimientos que les ayuden a cumplir con los objetivos; en este componente la 
información debe ser suficiente y relevante, además las actividades de control estarán diseñadas para un trabajo en conjunto entre departamentos. Posteriormente, dentro de la evaluación y el aprendizaje se debe analizar el control interno y los resultados en el logro de los objetivos precisando las diferencias encontradas para mejorar (Estupiñán y Niebel, 2015).

El marco integral de control interno para Latinoamérica (MICIL) es un modelo de control interno que al igual que los anteriores deberá ser conocido por todo el personal definiendo el rol de cada uno, su funcionamiento eficiente y eficaz para el logro de objetivos, la comunicación de la información obtenida en cada uno de los departamentos. Para que se lleve a cabo un control eficaz de las actividades de la empresa todas las personas deben estar apropiadas de éste y no solo encargarlo a un pequeño grupo de la organización. ( Pacheco, Narváez y Erazo, 2019)

EI MICIL deberá ser integrado en la organización primero con su diseño y luego con la evaluación de los resultados. Este modelo se aplica en pequeñas y medianas empresas "considerando los recursos humanos, materiales, financieros y tecnológicos administrados y evitando procedimientos complicados para las operaciones de la empresa" (Casals y Associates, 2004, p. 15). Para llevar a cabo la autoevaluación se recomienda realizarla en los primeros seis meses de operación para definir el grado de cumplimiento de objetivos, posteriormente esta autoevaluación se la realizará en cualquier momento y principalmente actividades que requieran una evaluación constante. Casals \& Associates (2004) afirman que al momento de realizar auditorias de estados financieros y de gestión es esencial la evaluación de este modelo de control interno.

Por otro lado, el modelo de control interno COSO ha sido creado para ayudar a la administración de las organizaciones en varios aspectos que garanticen la buena marcha de los negocios y la toma de decisiones (Santillana, Sistemas de Control Interno, 2015). Este modelo ha evolucionado desde 1992 (COSO I) hasta el 2013 (COSO III); mostrando un cambio evidente en sus componentes, pues, el COSO I elaborado en 1992 según Estupiñán y Niebel (2015) trabaja con 5 componentes que son:

- Ambiente de control 
- Evaluación de Riesgos

- Actividades de Control

- Información y Comunicación

- Supervisión y seguimiento

El COSO II del año 2005, por su parte se compone de:

- Ambiente interno

- Establecimiento de objetivos

- Identificación de eventos

- Evaluación de riesgos

- Respuesta al riesgo

- Actividades de control

- Información y comunicación

- Monitoreo

Y, finalmente el COSO III elaborado en el año 2013, tiene los siguientes componentes:

- Ambiente de control

- Evaluación de riesgos

- Actividades de control

- Información y comunicación

- Supervisión

Si bien el COSO I y COSO III tiene los mismos componentes, en este último se tiene una definición más exacta de trabajo de cada punto (Mantilla, 2013; Estupiñán y Niebel, 2015; Quinaluisa, Ponce, Muñoz, Ortega, y Pérez, 2018). El COSO III facilita el desarrollo, mantenimiento efectivo y eficiente de los sistemas de control y el fortalecimiento de las actividades para el logro de los objetivos (Santillana, Sistemas de Control Interno, 2015). En este sentido, se procede al análisis de cada uno de los componentes del modelo coso III.

1.- Ambiente de Control, contiene los procesos, estructuras y estándares que deben guiar al control interno en una organización. Siendo la gerencia o el equipo destinado a evaluar y controlar las actividades, quienes establecerán la importancia del control interno 
incluyendo en ellos los manuales de conducta. En este componente se encuentran los valores éticos de la empresa, los parámetros que seguirá la administración para el desempeño de la responsabilidad del gobierno, la estructura organizacional, responsabilidades del cargo, los procedimientos, entre otros. Los principios del ambiente de control del enfoque COSO son: la demostración del compromiso íntegro y ético, vigilar las responsabilidades, tener una estructura organizacional, autoridad y responsabilidad, compromiso del personal y la correcta asignación de responsabilidades (Mantilla, 2013; Santillana, 2015; Tapia, Guevara, Castillo, Rojas, y Salomón, 2016).

Blanco (2012); Tapia, Guevara, Castillo, Rojas, y Salomón, (2016) consideran algunos elementos que forman parte del ambiente de control; la comunicación y cumplimiento forzosos de la integridad y de los valores éticos, el compromiso por la competencia, la participación de quienes están a cargo de la gerencia, el estilo y filosofía de la administración, la estructura organizacional, la asignación de autoridad y responsabilidad y finalmente, las políticas y prácticas de recursos humanos. Mantilla (2013); Estupiñan y Niebel (2015); Quinaluisa N. , Ponce, Muñoz, Ortega, y Pérez, (2018) mencionan que el ambiente de control es el componente principal sobre el cual se basan los 4 componentes adicionales creando sus propios objetivos de control, este elemento se conforma de los valores éticos, la comunicación dentro de la organización, competencia, la junta directiva, la filosofía y el estilo de operación.

Como se evidencia en el análisis de este componente los autores tienen similitud en sus opiniones siendo para esta investigación los puntos clave que debe tener la Universidad y por ende cada departamento; un código de ética, un organigrama, el estilo de administración, la asignación de responsabilidades a cada miembro de la institución.

2.- Evaluación del riesgo, en este componente es importante analizar la probabilidad de ocurrencia y el impacto de cada uno de los aspectos o circunstancias que puedan alterar el buen funcionamiento de las actividades a desarrollarse en la institución. Por lo que es necesario el planteamiento de objetivos a cumplir por cada proceso o departamento, así se podrá identificar los riesgos relevantes para la consecución de los mismos, también 
se puede hacer uso de un mapa de riesgos (Mantilla, 2013; Sánchez, 2015; Serrano, Señalin, Vega, y Herrera, 2018).

3.- Actividades de Control, este componente se enfoca en las acciones que debe tomar la institución para verificar el cumplimiento de los procesos y procedimientos, de las normas legales y universitarias; para ello se debe establecer políticas, elaborar manuales de procesos y/o flujogramas que permitan conocer los pasos a seguir para ejecutar o desarrollar, en este caso, las tutorías académicas; es importante mencionar que a cada proceso y procedimiento se asigna una persona, de manera que se garantice el cumplimiento de los objetivos. Las actividades de control incluyen procesos de revisión y aprobacion en puntos estratégicos de la ejecución del sistema (Mantilla, 2013; Isaza, 2014; Tapia, Guevara, Castillo, Rojas, y Salomón, 2016; Quinaluisa N. , Ponce, Muñoz, Ortega, y Pérez, 2018).

4.- Información y comunicación, este elemento es necesario en todos los niveles y procesos de la institución, tanto los coordinadores como los tutores deben comunicarse lo que está ocurriendo, los cambios que se presentan a nivel interno y externo para tomar decisiones de manera oportuna que permitan cumplir con los objetivos y metas; además es importante que se tenga una buena comunicación con los beneficiarios y todos los involucrados en el proceso para que puedan direccionar hacia las personas indicadas y se continúe con el correcto desarrollo de los procedimientos (Mantilla, 2013; Tapia, Guevara, Castillo, Rojas, y Salomón, 2016).

5.- Supervisión y monitoreo, este al igual que los otros elementos del modelo COSO es importante en cada uno de los procesos y procedimientos o a nivel general para medir el cumplimiento de cada componente de control interno, esto puede ser mediante el análisis de indicadores. Así mismo se debe notificar los resultados encontrados en estas evaluaciones a los involucrados para buscar las soluciones y estrategias que ayuden a mitigar los riesgos de incumplimiento de los objetivos (Isaza, 2014; Santillana, 2015; Sulca y Becerra, 2017). 


\section{La Educación Superior en Ecuador}

La Constitución de la República del Ecuador expresa que la educación es un derecho de todas las personas y una prioridad para la inversión pública, garantizado la igualdad e inclusión. Señala además que se garantizará el acceso a la educación superior, la libertad de cátedra y el derecho se los padres a escoger para sus hijos la educación según sus principios y creencias. Por otra parte, menciona que la educación pública será gratuita y se garantizará la estabilidad de los docentes, salario justo, promoviendo el escalafón. En lo referente a la educación superior menciona que es una formación académica y profesional en cuanto a investigación científica, tecnológica y todas las competencias necesarias para resolver los problemas del país (Asamblea Nacional del Ecuador, 2008). Por su parte, la Ley Orgánica de Educación Superior (LOES) contempla en sus artículos lo referente a los derechos, fines, obligaciones, entre otros de todos los involucrados en la Educación Superior. En este ámbito se hará referencia a la calidad de educación que deberán brindar las Instituciones de Educación Superior (IES), siendo ésta un principio de la educación el cual consista en la búsqueda de mecanismos que fomente la autocrítica, la crítica externa y la mejora continua, también se abarca la evaluación de calidad contemplando algunos lineamientos que permitan acreditar a las IES en todos sus componentes, realizando un análisis cualitativo y cuantitativo, el mismo que deberá ser un proceso continuo en busca de un avance constante. El ente encargado de realizar las evaluaciones y de ubicar a las IES en una categoría es el Consejo de Evaluación, Acreditación y Aseguramiento de la Calidad de la Educación Superior (CEAACES) (Asamblea Nacional del Ecuador, 2010).

En este sentido, en el Ecuador se ha llevado acabo los procesos de evaluación y acreditación de las IES, contemplando cinco criterios de calificación; académico, organizativo, eficiencia académica, investigación e infraestructura (Grupo El Universo, 2013), luego del analisis se procede a dar una calificación A, B, C O D (Ecuador Goverment, 2013).

Siendo esta una de las razones para que las IES tomen acciones de cambio y una visión hacia la ubicación en la primera categoría, sin embargo, ha existido quienes no han 
podido obtener una evaluación aceptable y han procedido al cierre de las mismas, dejando con las carreras inconclusas a los jóvenes, quienes tuvieron que convalidar materias en otras universidades para continuar sus estudios. Por todo lo señalado anteriormente, se ha creado la Secretaría Nacional de Educación Superior, Ciencia, Tecnología e Innovación (SENESCYT) cuyo objetivo es "promover la formación del talento humano avanzado y el desarrollo de la investigación, innovación y transferencia tecnológica" (SENESCYT, 2018), creando así un sistema de asignación de los estudiantes que postulen para estudiar un tercer nivel, teniendo que registrarse y seleccionar las carreras en orden de su preferencia, una vez rendido el examen se les ubica en las diferentes universidades del país.

Sin embargo, esto no ha sido visto con buenos ojos por los jóvenes pues muchas veces han sido asignados en carreras que no son de su agrado (Grupo El Universo, 2014). Esto ocaciona que los estudiantes sientan desmotivacion en los estudios y sigan la carrera unicamente por cumplir con la sociedad, siendo una de las causas primodiales de la existencia de un alto indice de desercion y repitencia en las IES; esto esta ligado también con las situaciones personales que complican su rendimiento, además que el definir si los jovenes tienen un bueno rendimiento o no se dificulta en la educación superior, pues tienen más alternativas para gestionar su carrera como, al poder elegir las materias a cursar los jóvenes pueden adelantar o simplemente no coger en ese ciclo la asignatura, lo que impide determinar con exactitud un buen o mal resultado (Martín, García, Torbay, y Rodríguez, 2008).

Complementando al criterio de estos autores, se reconocen otros factores como familiares, económicos, sociales, culturales, académicos, la capacidad de autoaprendizaje, los conocimiento básicos, entre otros, que influyen en los jóvenes durante sus estudios universitarios, los mismos que se evidencia principalmente en los primeros ciclos (Jara, y otros, 2008). Siendo estas las razones por las cuales las universidades han buscado un mecanismo para reducir los indices de decersion y repitencia, siendo uno de estos las tutorías universitarias, pero para poder evaluar la 
calidad y la correcta aplicación de esta propuesta es importante la aplicación de un sistema de control interno en todos sus procesos y procedimientos.

\section{METODOLOGÍA}

El diseño empleado en este estudio fue "no experimental" pues ha sido basado en el análisis de la situación actual para, con la ayuda del estudio de la literatura, poder plantear una solución, el enfoque fue mixto porque se realizaron análisis cuantitativos a través de encuestas y entrevistas a los involucrados, también se realizó la investigación teórica y situacional de las variables en la actualidad, siendo este el estudio cualitativo lo que permitió conocer de manera amplia el tema de investigación agregando valor a la misma. El alcance fue descriptivo debido a que se buscó crear un sistema de control interno para una institución de educación superior basado en la información que nos brinda la literatura de estudios realizados anteriormente.

La finalidad del estudio se lo realizó de manera transversal debido a que se analizaron los acontecimientos ocurridos en un solo momento de tiempo, los métodos empleados fueron: histórico-lógico, inductivo-deductivo, analítico-sintético, estadístico y sistémico, los mismos que se emplearon a lo largo de toda la investigación para definir una propuesta con criterios lógicos y reales. Las técnicas empleadas fueron: encuestas, entrevista, observación y análisis documental, llegando a utilizar instrumentos como el cuestionario, la guía de preguntas, guía de observación y la ficha de evaluación documental, respectivamente. La unidad de análisis fue el Departamento de Pastoral de la Universidad Politécnica Salesiana determinando como involucrados a 21 estudiantes tutores, 1 coordinador y 1 Director Técnico Departamental, siendo la población de 23 personas. 


\section{RESULTADOS}

Luego de la aplicación de la metodología indicada anteriormente se obtuvieron los siguientes resultados:

- El Departamento de Pastoral Universitaria es parte importante de la estructura organizativa de la Universidad Politécnica Salesiana, pues trabaja en el eje transversal de la formación de los futuros profesionales del país.

- La acción de las tutorías entre pares realizada por estudiantes hacia otros estudiantes es una propuesta relevante para el departamento y por su puesto para la institución.

- La estructura que tiene la propuesta de tutorías académicas es deficiente, pues al no estar normados los procesos se ha estado impartiendo de manera empírica y generando que los estudiantes creen su propia metodología de enseñanza.

- Se ha realizado una sola vez una encuesta de satisfacción a los estudiantes que recibieron la tutoría, lo que no permite que se evidencia período a período si es correcta y de calidad la tutoría brindada por estudiantes.

- No existen fichas de seguimiento por estudiantes que permitan analizar la evolución que han tenido y dar seguimiento al proceso de aprendizaje, sino hasta el final del período académico, lo que es perjudicial para los tutorados.

- El Departamento de Pastoral no cuenta con manuales y reglamentos de tutoría aprobados; reportes de entrega de informes; fichas de seguimiento a los estudiantes que reciben la tutoría y de resultados obtenidos; ni informes de encuestas de satisfacción.

- No hay una persona encargada de verificar que las tutorías se estén realizando en el día y la hora establecida en los horarios, por lo que no se tiene la certeza de que la tutoría sea impartida.

- Los tutores no entregan un informe de las tutorías impartidas al final del mes.

- La mayoría de involucrados están conscientes que con la implementación de un sistema de control interno se mejorarían los procesos y procedimientos de las tutorías brindas por estudiantes a estudiantes. 


\begin{tabular}{|c|c|}
\hline \multirow{2}{*}{$\begin{array}{c}\text { UNIVERSIDAD POLITÉCNICA } \\
\text { SALESIANA }\end{array}$} & UNIVERSIDAD POLITÉCNICA SALESIANA \\
\cline { 2 - 2 } & PROCEDIMIENTOS DE CONTROL INTERNO \\
\hline
\end{tabular}

\begin{tabular}{|c|c|c|c|c|c|}
\hline $\mathbf{N}^{\circ}$ & PROCESO & OBJETIVO & PROCEDIMIENTO & CONTROL & RIESGOS \\
\hline \multirow{4}{*}{1} & \multirow{4}{*}{$\begin{array}{c}\text { Contratación de } \\
\text { tutores pares }\end{array}$} & \multirow{4}{*}{$\begin{array}{c}\text { Comprobar } \\
\text { que el proceso } \\
\text { de } \\
\text { contratación } \\
\text { se cumpla }\end{array}$} & $\begin{array}{l}\text { Identificar la } \\
\text { necesidad de } \\
\text { tutores. }\end{array}$ & $\begin{array}{l}\text { Verificar documentación } \\
\text { que emite el Director } \\
\text { Departamental a la } \\
\text { autoridad competente } \\
\text { sobre la necesidad de } \\
\text { contratar estudiantes } \\
\text { tutores. }\end{array}$ & $\begin{array}{l}\text { No exista el personal } \\
\text { comprometido con la } \\
\text { coordinación que permita } \\
\text { cumplir con el desarrollo } \\
\text { de las tutorías y la } \\
\text { elaboración de informes } \\
\text { de resultados. }\end{array}$ \\
\hline & & & $\begin{array}{l}\text { Establecer } \\
\text { competencias de } \\
\text { los tutores. }\end{array}$ & $\begin{array}{l}\text { Verificar proyecto en } \\
\text { donde esté incluido las } \\
\text { competencias que deben } \\
\text { cumplir los tutores. }\end{array}$ & $\begin{array}{l}\text { Seleccionar a quienes no } \\
\text { tengan las capacidades } \\
\text { para ser tutores pares. }\end{array}$ \\
\hline & & & $\begin{array}{l}\text { Selección de } \\
\text { tutores. }\end{array}$ & $\begin{array}{l}\text { Verificar el informe del } \\
\text { proceso de análisis y } \\
\text { búsqueda de estudiantes } \\
\text { tutores con las firmas del } \\
\text { director del Departamento } \\
\text { y el Coordinador de } \\
\text { Tutorías. }\end{array}$ & $\begin{array}{l}\text { Decidir unilateralmente a } \\
\text { los estudiantes tutores. }\end{array}$ \\
\hline & & & $\begin{array}{l}\text { Aprobación para } \\
\text { contratación. }\end{array}$ & $\begin{array}{l}\text { Verificar correo/oficio de } \\
\text { aprobación de }\end{array}$ & $\begin{array}{l}\text { No tener personal } \\
\text { comprometido para el } \\
\text { desarrollo de las tutorías. }\end{array}$ \\
\hline
\end{tabular}


Revista Arbitrada Interdisciplinaria KOINONIA

Año IV. Vol IV. ํ⒉ Edición especial 2019

Hecho el depósito de Ley: FA2016000010

ISSN: 2542-3088

FUNDACIÓN KOINONIA (F.K). Santa Ana de Coro. Venezuela.

Erika Priscila Zamora Cabrera; Cecilia Ivonne Narváez Zurita; Juan Carlos Erazo Álvarez

\begin{tabular}{|l|l|l|l|l|}
\hline & & & $\begin{array}{l}\text { contratación por el } \\
\text { Vicerrector. }\end{array}$ & \\
\hline
\end{tabular}




\section{Contratación de tutores}

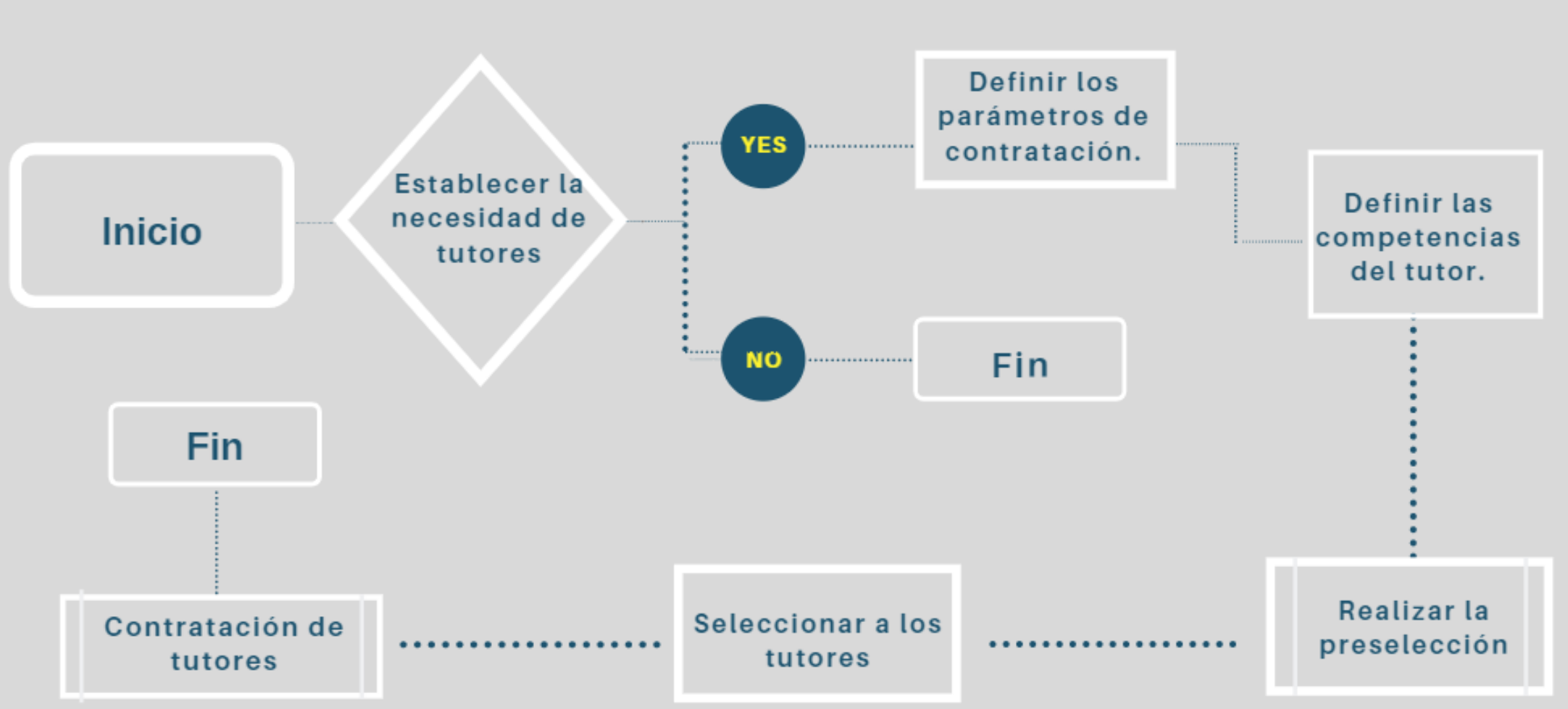




\begin{tabular}{|c|c|}
\hline \multirow{3}{*}{$(4-1)$ UNIVERSIDAD POLITÉCNICA } & UNIVERSIDAD POLITÉCNICA SALESIANA \\
\hline & PROCEDIMIENTOS DE CONTROL INTERNO \\
\hline & Tutorías académicas \\
\hline
\end{tabular}

\begin{tabular}{|c|c|c|c|c|c|}
\hline $\mathbf{N}^{\circ}$ & PROCESO & OBJETIVO & PROCEDIMIENTO & CONTROL & RIESGOS \\
\hline \multirow{6}{*}{2} & \multirow{6}{*}{$\begin{array}{l}\text { Tutorías } \\
\text { académicas }\end{array}$} & \multirow{6}{*}{$\begin{array}{c}\text { Comprobar que } \\
\text { en los procesos } \\
\text { de las tutorías se } \\
\text { tenga } \\
\text { documentación } \\
\text { que garantice la } \\
\text { buena marcha de } \\
\text { las tutorías. }\end{array}$} & $\begin{array}{l}\text { Recepción de base } \\
\text { de datos de nuevos } \\
\text { becarios. }\end{array}$ & $\begin{array}{l}\text { Verificar documento entrega - } \\
\text { recepción de base de datos } \\
\text { con las respectivas firmas de } \\
\text { respaldo. }\end{array}$ & $\begin{array}{l}\text { No poder acercarse a los } \\
\text { jóvenes becarios que necesitan } \\
\text { acompañamiento. }\end{array}$ \\
\hline & & & $\begin{array}{l}\text { Consolidación } \\
\text { nuevos y antiguos } \\
\text { becarios. }\end{array}$ & $\begin{array}{l}\text { Verificar documento } \\
\text { consolidado de beneficiarios } \\
\text { de tutoría. }\end{array}$ & $\begin{array}{l}\text { No conocer la realidad de los } \\
\text { becarios en la universidad y su } \\
\text { proceso educativo. }\end{array}$ \\
\hline & & & $\begin{array}{l}\text { Planificación de los } \\
\text { horarios de tutoría. }\end{array}$ & $\begin{array}{l}\text { Verificar ficha de horarios con } \\
\text { firmas de revisión y aprobación }\end{array}$ & $\begin{array}{l}\text { Seleccionar horarios en los } \\
\text { cual no se tenga asistencia de } \\
\text { los beneficiarios. }\end{array}$ \\
\hline & & & $\begin{array}{l}\text { Socialización de la } \\
\text { tutoría y horarios. }\end{array}$ & $\begin{array}{l}\text { Verificar que los registros de } \\
\text { asistencia cuenten con todos } \\
\text { los datos solicitados y las } \\
\text { respectivas firmas de los } \\
\text { beneficiarios. }\end{array}$ & $\begin{array}{l}\text { No tener evidencia que } \\
\text { sustente la tutoría lo cual } \\
\text { podría ser motivo de cierre del } \\
\text { proceso. }\end{array}$ \\
\hline & & & $\begin{array}{l}\text { Aplicación de la } \\
\text { tutoría }\end{array}$ & $\begin{array}{l}\text { Verificar las fichas de } \\
\text { seguimiento con las } \\
\text { respectivas firmas. }\end{array}$ & $\begin{array}{l}\text { Desconocer cuál es la } \\
\text { evolución que ha tenido el } \\
\text { estudiante durante el proceso y } \\
\text { por ende se puede errar al } \\
\text { emitir criterios de su estado } \\
\text { académico y personal. }\end{array}$ \\
\hline & & & $\begin{array}{l}\text { Elaboración de } \\
\text { informes semanales } \\
\text { y mensuales. } \\
\text { Entrega de informes } \\
\text { al coordinador. }\end{array}$ & $\begin{array}{l}\text { Verificar que el documento } \\
\text { entrega - recepción de } \\
\text { informes contenga las firmas } \\
\text { respectivas de los } \\
\text { responsables. }\end{array}$ & $\begin{array}{l}\text { No poder garantizar que la } \\
\text { información con la cual se } \\
\text { presenta resultados sea real y } \\
\text { completa. }\end{array}$ \\
\hline
\end{tabular}




\section{Tutorías académicas}

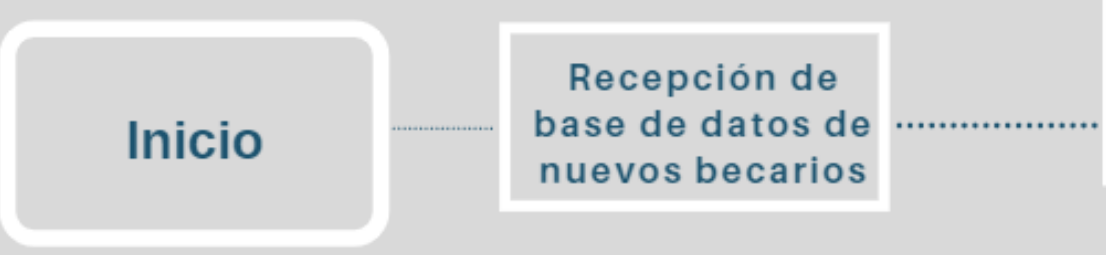

\section{Consolidación de nuevos y antiguos becarios.}

\section{Fin}

\section{Entrega de informes al Coordinadory Director} departamental

\section{Elaboración} de informes
Preparación de horarios

Socialización de la tutoría académica

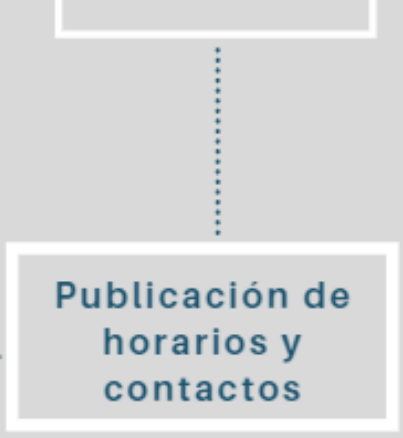




\begin{tabular}{|c|c|}
\hline \multirow{2}{*}{ UNIIERSIDAD POLITÉCNICA } & UNIVERSIDAD POLITÉCNICA SALESIANA \\
\cline { 2 - 2 } SALESIANA A A & PROCEDIMIENTOS DE CONTROL INTERNO \\
\cline { 2 - 3 } & Elaboración de informes \\
\hline
\end{tabular}

\begin{tabular}{|c|c|c|c|c|c|}
\hline $\mathbf{N}^{\circ}$ & PROCESO & OBJETIVO & PROCEDIMIENTO & CONTROL & RIESGOS \\
\hline \multirow{5}{*}{3} & \multirow{5}{*}{$\begin{array}{c}\text { Elaboración de } \\
\text { informes }\end{array}$} & \multirow{5}{*}{$\begin{array}{l}\text { Verificar que el } \\
\text { procesamiento de } \\
\text { la información } \\
\text { para la } \\
\text { presentación de } \\
\text { resultados sea } \\
\text { correcto. }\end{array}$} & $\begin{array}{l}\text { Receptar } \\
\text { informes } \\
\text { semanales y } \\
\text { mensuales. }\end{array}$ & $\begin{array}{l}\text { Verificar documento } \\
\text { entrega - recepción de } \\
\text { informes semanales y } \\
\text { mensuales con las } \\
\text { respectivas firmas que } \\
\text { validen la información. }\end{array}$ & $\begin{array}{l}\text { No garantizar la } \\
\text { recepción de los } \\
\text { informes. }\end{array}$ \\
\hline & & & $\begin{array}{l}\text { Establecer } \\
\text { indicadores. }\end{array}$ & $\begin{array}{l}\text { Verificar la existencia de } \\
\text { un documento que } \\
\text { contenga los indicadores } \\
\text { a evaluar de las tutorías } \\
\text { académicas. }\end{array}$ & $\begin{array}{l}\text { No presentar los } \\
\text { resultados en todas las } \\
\text { áreas. }\end{array}$ \\
\hline & & & $\begin{array}{l}\text { Elaborar gráficos } \\
\text { estadísticos. }\end{array}$ & $\begin{array}{l}\text { Verificar correo/oficio en } \\
\text { el cual se delegue a un } \\
\text { tutor para la validación de } \\
\text { la información presentada } \\
\text { en los indicadores. }\end{array}$ & $\begin{array}{l}\text { Presentación errónea } \\
\text { de resultados. }\end{array}$ \\
\hline & & & $\begin{array}{l}\text { Revisar la } \\
\text { información } \\
\text { obtenida en base } \\
\text { a gráficos. }\end{array}$ & $\begin{array}{l}\text { Verificar informes de } \\
\text { validación de resultados } \\
\text { presentados en los } \\
\text { indicadores, con las } \\
\text { respectivas firmas. }\end{array}$ & $\begin{array}{l}\text { No hay garantía de la } \\
\text { veracidad de } \\
\text { resultados. }\end{array}$ \\
\hline & & & $\begin{array}{l}\text { Socialización de } \\
\text { resultados. }\end{array}$ & $\begin{array}{l}\text { Verificar registros de } \\
\text { asistencia a las }\end{array}$ & $\begin{array}{l}\text { Reclamos desde las } \\
\text { autoridades por la falta }\end{array}$ \\
\hline
\end{tabular}


Revista Arbitrada Interdisciplinaria KOINONIA

Año IV. Vol IV. ํ⒉ Edición especial 201

Hecho el depósito de Ley: FA2016000010

ISSN: 2542-3088

FUNDACIÓN KOINONIA (F.K). Santa Ana de Coro. Venezuela.

Erika Priscila Zamora Cabrera; Cecilia Ivonne Narváez Zurita; Juan Carlos Erazo Álvarez

\begin{tabular}{|l|l|l|l|l|}
\hline & & & $\begin{array}{l}\text { socializaciones con las } \\
\text { firmas de los asistentes. }\end{array}$ & $\begin{array}{l}\text { de socializaciones } \\
\text { periódicas. }\end{array}$ \\
\hline
\end{tabular}




\section{Elaboración de informes}

Recepción de

informes

semanales y

mensuales

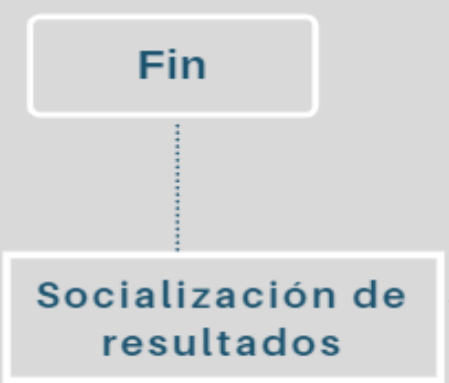

La información es correcta
Establecer

indicadores
Procesar

información

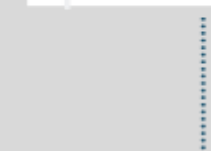

Elaborar gráficos estadísticos 


\section{Discusión y conclusiones}

Las organizaciones se componen de departamentos que se encargan de ejecutar las acciones necesarias para que se logren los objetivos por varias ramas. Para ello existen procesos que al tener un buen control permitirán que se cumpla con los deseos de todos los que conforman la institución.

La falta de controles en las diversas actividades que se ejecutan en los procesos provocan que no se logren los objetivos de manera eficiente, eficaz y económico.

Por lo tanto, las instituciones, al incorporar procedimientos de control interno en todos sus procesos puede controlar y evaluar constantemente el correcto funcionamiento de los procesos que le permitirán llegar a conseguir sus metas mitigando los posibles riesgos que se generen en la puesta en marcha de sus actividades, siendo esto pertinente con lo planteado por Colina (2017).

El tener varios modelos de control interno, permiten tener guías para la implementación de controles internos en las diferentes instituciones y para todas las estructuras.

El control interno incide rotundamente en la gestión administrativa, pues permite que los procesos sean eficientes, efectivos y económicos; pues la institución se apoya en estos procedimientos para garantizar la permanencia y grado de sus estudiantes, cumpliendo con las políticas y normas legales y universitarias, además de los criterios de evaluación del Consejo de Aseguramiento de la Calidad de la Educación Superior (CACES). 


\section{REFERENCIAS CONSULTADAS}

1. Asamblea Nacional del Ecuador. (2008). Constitución de la República del Ecuador. Quito. Recuperado el 21 de junio de 2018, de https://www.oas.org/juridico/pdfs/mesicic4 ecu const.pdf

2. Asamblea Nacional del Ecuador. (2010). LEY ORGANICA DE EDUCACION SUPERIOR, LOES. Quito: Lexis.

3. Bartolomé, S. (2018). Manual. Organización empresarial y de RRHH (UF0517). Certificados de profesionalidad. Operaciones auxiliares de servicios administrativos y generales (ADGG0408). Madrid: CEP.

4. Benavides Ortega, R., Narváez Zurita, C., Erazo Álvarez, J., \& Ordoñez Parra, Y. (junio de 2019). Auditoría financiera como herramienta de análisis de los estados financieros de la empresa Imgrumasa S.A. de la ciudad de Machala, periodo 2018. Visionario Digital, 577-598. doi:https://doi.org/10.33262/visionariodigital.v3i2.1.589

5. Colina, M. (2017). Ambiente virtual de aprendizaje de contabilidad ii para las horas de trabajo independiente del programa nacional de formación en administración. Revista Arbitrada Interdisciplinaria Koinonía, 2(3), 92-108. Recuperado de http://fundacionkoinonia.com.ve/ojs/index.php/revistakoinonia/article/view/54/41

6. Ecuador Goverment. (27 de 11 de 2013). Solo tres universidades se ubican en categoría A. Recuperado el 22 de 12 de 2017, de http://www.eltelegrafo.com.ec/noticias/sociedad/4/solo-tres-universidades-seubican-en-categoria-a

7. Fonseca, O. (2013). Sistemas de Control Interno Para Organizaciones. Lima: IICO. Obtenido https://books.google.com.ec/books?id=plsiU8xoQ9EC\&pg=PA26\&dq=cobit+mod elo+de+control+interno\&hl=es-419\&sa $=$ X\&ved=0ahUKEwiLm9KI3LaAhXP21MKHaHnAHYQ6AEIJjAA\#v=onepage\&q=cobit\%20modelo\%20de\%20 control\%20interno\&f=false

8. Gamboa, J., Puente, S., \& Vera, P. (2016). Importancia del control interno en el sector público. Publicando, 3(8), 487-502. Recuperado el 09 de agosto de 2019

9. Grupo El Universo. (30 de 11 de 2013). 46 indicadores en 5 categorías para evaluar a las universidades del país. Recuperado el 22 de 12 de 2017, de https://www.eluniverso.com/noticias/2013/11/30/nota/1849901/46-indicadores-5categorias-evaluar-universidades-pais 
10. Grupo El Universo. (30 de 11 de 2014). En dos años disminuyó el ingreso a la universidad en Ecuador. Recuperado el 19 de Junio de 2018, de https://www.eluniverso.com/noticias/2014/11/30/nota/4284736/dos-anosdisminuyo-ingreso-universidad

11. Isaza, A. (2014). Control Interno y Sistema de Gestión de Calidad (2da. ed.). Bogotá, Colombia: Ediciones de la U. Recuperado el 29 de junio de 2018

12. Jara, D., Valverde, H., Gordillo, G., Guerra, G., León, I., Arroyo, C., \& Figueroa, M. (2008). Factores influyentes en el rendimiento académico de estudiantes del primer año de medicina. Anales de la Facultad de Medicina, 69(3), 193-197. Recuperado el 21 de junio de 2018, de http://www.scielo.org.pe/scielo.php?pid=S1025$\underline{55832008000300009 \& s c r i p t=s c i}$ arttext

13. López, A., \& Pesántez, J. (enero - abril de 2017). Evaluación comparativa del sistema de control interno del sector comercial y del sector público del Cantón Morona. Killkana Sociales, 1(1), 31-38. Recuperado el 9 de agosto de 2019, de https://dialnet.unirioja.es/servlet/articulo?codigo $=6297494$

14. Mantilla, S. (2013). Auditoría de control interno (Vol. tercera). Bogotá, Colombia: Ecoe Ediciones. Recuperado el 09 de agosto de 2019, de https://books.google.com.ec/books?id=rMS4DQAAQBAJ\&printsec=frontcover\&hl =es\#v=onepage \&q\&f=false

15. Mendívil, V. (2016). Elementos de Auditoría (7ma ed.). México D.F., México: Cengage Learning Editoes S.A. Recuperado el 09 de agosto de 2019

16. Pacheco Poma, W. P., Narváez Zurita, C. I., \& Erazo Álvarez, J. C. (2019). Procedimiento metodológico de auditoría interna para la Cooperativa de Ahorro y Crédito Santiago Ltda., del cantón Loja. Visionario Digital, 313.

17. Quinaluisa, N., Ponce, V., Muñoz, S., Ortega, X., \& Pérez, J. (ene-jun de 2018). El control interno y sus herramientas de aplicación entre COSO y COCO. (C. Habana, Ed.) Scielo, 12(1), 268-283. Recuperado el 09 de agosto de 2019, de http://scielo.sld.cu/scielo.phd?script=sci arttext\&pid=S2073$\underline{60612018000100018 \& \operatorname{lng}=e s \& \text { thng=es }}$

18. Quinaluisa, N., Ponce, V., Muñoz, S., Ortega, X., \& Pérez, J. (2018). El control interno y sus herramientas de aplicación entre COSO y COCO. Cofin Habana, 12(1), 268-283. Recuperado el 10 de Agosto de 2019, de http://scielo.sld.cu/pdf/cofin/v12n1/cofin18118.pdf 
19. Sánchez, L. (2015). COSO ERM y la gestión de riesgos. QUIPUKAMAYOC, 23(44), 43-50. Recuperado el 10 de agosto de 2019, de file:///C:/Users/User/Downloads/11625-Texto\%20del\%20art\%C3\%ADculo-405841-10-20160330.pdf

20. Santillana, J. (2015). Sistemas de control interno (3ra ed.). México, México: Pearson Educación. Recuperado el 10 de agosto de 2019

21. Santillana, J. (2015). Sistemas de Control Interno (3ra. ed.). México D.C., México: Pearson Educación. Recuperado el 29 de junio de 2018

22. SENESCYT. (2014). Secretaría de Educación Superior Ciencia, Tecnología e Innovación. Recuperado el 14 de 12 de 2017, de http://www.educacionsuperior.gob.ec/en-azuay-la-gestion-en-educacion-superiorcumple-y-alienta/

23. SENESCYT. (2018). Secretaría de Educación Superior, Ciencia, Tecnología e Innovación. Recuperado el 21 de junio de 2018, de https://www.educacionsuperior.gob.ec/valores-mision-vision/

24. Serrano, P., Señalin, L., Vega, F., \& Herrera, J. (2018). El control interno como herramienta indispensable para una gestion financiera y contable eficiente en las empresas bananeras del cantón Machcala. Espacios, 39(3), 30. Recuperado el 10 de agosto de 2019, de http://www.revistaespacios.com/a18v39n03/a18v39n03p30.pdf

25. Sulca, G., \& Becerra, E. (2017). Control interno. Matriz de riesgo: Aplicación metodológica COSO II. Publicando, 4(12), 106-125. Recuperado el 10 de agosto de 2019, de https://revistapublicando.org/revista/index.php/crv/article/view/686/pdf 491

26. Tapia, C., Guevara, E., Castillo, S., Rojas, M., \& Salomón, L. (2016). Fundamentos de Auditoría (1ra ed.). Mexico: Instituto Mexicano de Contadores Públicos. Recuperado el 9 de agosto de 2019

27. Universidad Politécnica Salesiana. (2015). Programa de acompañamiento salesiano a usuarios de la política de cuotas para la Educación Superior (PASUPCES). Resolución Consejo Superior, Cuenca. Recuperado el 21 de junio de 2018 , de https://www.ups.edu.ec/documents/10184/20838/Programa+acompa\%C3\%B1am iento+salesiano+a+usuarios+pol\%C3\%ADtica+de+cuotas+para+la+educaci\%C3 \%B3n+superior/d65743e9-3f5b-46d2-87ae-2943a59f4608 


\section{REFERENCES CONSULTED}

1. National Assembly of Ecuador. (2008). Constitution of the Republic of Ecuador. Quito Retrieved on June 21, 2018, from https://www.oas.org/juridico/pdfs/mesicic4 ecu const.pdf

2. National Assembly of Ecuador. (2010). ORGANIC LAW OF HIGHER EDUCATION, LOES. Quito: Lexis.

3. Bartolomé, S. (2018). Manual. Business and HR Organization (UF0517). Professional certificates. Auxiliary operations of administrative and general services (ADGG0408). Madrid: CEP.

4. Benavides Ortega, R., Narváez Zurita, C., Erazo Álvarez, J., \& Ordoñez Parra, Y. (June 2019). Financial audit as a tool for analyzing the financial statements of the company Imgrumasa S.A. from the city of Machala, 2018 period. Digital Visionary, 577-598. doi: https: //doi.org/10.33262/visionariodigital.v3i2.1.589

5. Colina, M. (2017). Virtual accounting learning environment ii for working hours independent of the national administration training program. Interdisciplinary Arbitrated Review Koinonía, 2 (3), 92-108. Recovered from http://fundacionkoinonia.com.ve/ojs/index.php/revistakoinonia/article/view/54/41

6. Ecuador Goverment. (27 of 11 of 2013). Only three universities are located in category A. Retrieved on December 22, 2017, from http://www.eltelegrafo.com.ec/noticias/sociedad/4/solo-tres-universidades-seubican-en-categoria-to

7. Fonseca, O. (2013). Internal Control Systems For Organizations. Lima: IICO. Retrieved https://books.google.com.ec/books?id=plsiU8xoQ9EC\&pg=PA26\&dq=cobit+mod elo+de+control+interno\&hl=en-419\&sa=X\&ved=0ahUKEwiLm9KI3LaAhXP21MKHaHnAHYQ6AElj\%\&qqmode $===20 \%$ control20\%internal\&f=false

8. Gamboa, J., Puente, S., \& Vera, P. (2016). Importance of internal control in the public sector. Publishing, 3 (8), 487-502. Retrieved on August 09, 2019

9. The Universe Group. (30 of 11 of 2013). 46 indicators in 5 categories to evaluate the country's universities. Retrieved on December 22, 2017, from https://www.eluniverso.com/noticias/2013/11/30/nota/1849901/46-indicadores-5categorias-evaluar-universidades-pais

10. Group The Universe. (30 of 11 of 2014). In two years the entrance to the university in Ecuador decreased. Retrieved on June 19, 2018, from 
https://www.eluniverso.com/noticias/2014/11/30/nota/4284736/dos-anosdisminuyo-ingreso-universidad

11. Isaza, A. (2014). Internal Control and Quality Management System (2nd ed.). Bogotá, Colombia: Editions of the U. Retrieved on June 29, 2018

12. Jara, D., Valverde, H., Gordillo, G., Guerra, G., León, I., Arroyo, C., \& Figueroa, M. (2008). Influential factors in the academic performance of first year medical students. Annals of the Faculty of Medicine, 69 (3), 193-197. Retrieved on June 21, 2018, from http://www.scielo.org.pe/scielo.php?pid=S1025$\underline{55832008000300009 \& \text { script }=\text { sci arttext }}$

13. López, A., \& Pesántez, J. (January - April 2017). Comparative evaluation of the internal control system of the commercial sector and the public sector of Canton Morona. Killkana Social, 1 (1), 31-38. Retrieved on August 9, 2019, from https://dialnet.unirioja.es/servlet/articulo?codigo $=6297494$

14. Mantilla, S. (2013). Internal control audit (Vol. Third). Bogotá, Colombia: Ecoe Editions. Retrieved on August 9, 2019, from https://books.google.com.ec/books?id=rMS4DQAAQBAJ\&printsec=frontcover\&hl $=e n \# v=0 n e p a g e \& q \& f=$ false

15. Mendívil, V. (2016). Elements of Audit (7th ed.). México D.F., Mexico: Cengage Learning Editoes S.A. Retrieved on August 09, 2019

16. Pacheco Poma, W. P., Narváez Zurita, C. I., \& Erazo Álvarez, J. C. (2019). Internal audit methodological procedure for the Cooperativa de Ahorro y Crédito Santiago Ltda., Of the Loja canton. Digital Visionary, 313.

17. Quinaluisa, N., Ponce, V., Muñoz, S., Ortega, X., \& Pérez, J. (Jan-Jun 2018). Internal control and its application tools between COSO and COCO. (C. Habana, Ed.) Scielo, 12 (1), 268-283. Retrieved on August 9, 2019, from http://scielo.sld.cu/scielo.phd?script=sci arttext\&pid=S2073-

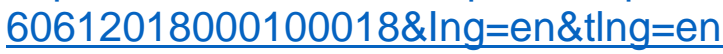

18. Quinaluisa, N., Ponce, V., Muñoz, S., Ortega, X., \& Pérez, J. (2018). Internal control and its application tools between COSO and COCO. Cofin Habana, 12 (1), 268-283. Retrieved on August 10, 2019, from http://scielo.sld.cu/pdf/cofin/v12n1/cofin18118.pdf

19. Sánchez, L. (2015). COSO ERM and risk management. QUIPUKAMAYOC, 23 (44), 43-50. Retrieved on August 10, 2019, from 
file:///C:/Users/User/Downloads/11625-Texto\%20del\%20art\%C3\%ADculo-405841-10-20160330.pdf

20. Santillana, J. (2015). Internal control systems (3rd ed.). Mexico, Mexico: Pearson Education. Retrieved on August 10, 2019.

21.Santillana, J. (2015). Internal Control Systems (3rd ed.). Mexico D.C., Mexico: Pearson Education. Retrieved on June 29, 2018

22. SENESCYT. (2014). Ministry of Higher Education Science, Technology and Innovation. Retrieved on December 14, 2017, from http://www.educacionsuperior.gob.ec/en-azuay-la-gestion-en-educacion-superiorcumple-y-alienta/

23. SENESCYT. (2018). Ministry of Higher Education, Science, Technology and Innovation. Retrieved on June 21, 2018, from https://www.educacionsuperior.gob.ec/valores-mision-vision/

24. Serrano, P., Signoin, L., Vega, F., \& Herrera, J. (2018). Internal control as an indispensable tool for efficient financial and accounting management in banana companies in the Machcala canton. Spaces, 39 (3), 30. Retrieved on August 10, 2019, from http://www.revistaespacios.com/a18v39n03/a18v39n03p30.pdf

25. Sulca, G., \& Becerra, E. (2017). Internal control. Risk matrix: COSO II methodological application. Publishing, 4 (12), 106-125. Retrieved on August 10, 2019, https://revistapublicando.org/revista/index.php/crv/article/view/686/pdf 491

26. Tapia, C., Guevara, E., Castillo, S., Rojas, M., \& Salomón, L. (2016). Fundamentals of Audit (1st ed.). Mexico: Mexican Institute of Public Accountants. Retrieved on August 9, 2019

27. Salesian Polytechnic University. (2015). Salesian support program for users of the Quota Policy for Higher Education (PASUPCES). Superior Council Resolution, Cuenca. Retrieved on June 21, 2018, from https://www.ups.edu.ec/documents/10184/20838/Program+acompa\%C3\%B1ami ento+salesiano+a+ustarios+pol\%C3\%ADtica+de+quotas+for+the+education\%C3 \%B3n+higher/d65743e9-3f5b-46d2-87ae-2943a59f4608

(C2019 por el autor. Este artículo es de acceso abierto y distribuido según los términos y condiciones de la licencia Creative Commons Atribución-NoComercial-Compartirlgual 4.0 Internacional (CC BY-NC-SA 4.0) (https://creativecommons.org/licenses/by-nc-sa/4.0/). 